\title{
A Novel Hybrid MPPT for Wind Energy Conversion Systems Operating under Low Variations in Wind Speed
}

\author{
Paul Abena Malobe1, Philippe Djondine'1,2*, Pascal Ntsama Eloundou1, Hervé Abena Ndongo1 \\ ${ }^{1}$ Department of Physics, Faculty of Science, The University of Ngaoundéré, Ngaoundéré, Cameroon \\ ${ }^{2}$ Department of Physics, Higher Teacher Training College of Bertoua, The University of Ngaoundéré, Bertoua, Cameroon \\ Email: *pdjondine@yahoo.fr
}

How to cite this paper: Malobe, P.A., Djondine, P., Eloundou, P.N. and Ndongo, H.A. (2020) A Novel Hybrid MPPT for Wind Energy Conversion Systems Operating under Low Variations in Wind Speed. Energy and Power Engineering, 12, 716-728. https://doi.org/10.4236/epe.2020.1212042

Received: October 21, 2020

Accepted: December 28, 2020

Published: December 31, 2020

Copyright $\odot 2020$ by author(s) and Scientific Research Publishing Inc. This work is licensed under the Creative Commons Attribution International License (CC BY 4.0).

http://creativecommons.org/licenses/by/4.0/

\section{(c) (i) Open Access}

\begin{abstract}
This manuscript presents a new approach MPPT (Maximum Power Point Tracking) for improving and optimizing the performance of a Wind Energy Conversion System (WECS) operating for small variations in wind speed by combining sliding mode control and fuzzy logic control. The proposed method consists of optimizing the sliding mode controller by the fuzzy controller. The main purpose of the Sliding Mode control-Fuzzy Logic controller (SM-FL) is to ensure the robustness (by eliminating certain disadvantages of the sliding mode control such as the phenomenon of chattering) and the stability of the control system in the case of small variations in conditions atmospheric (here variation of the wind). Our system consists of a wind turbine, a Permanent Magnet Synchronous Generator (PMSG) and a DC-DC boost converter connected to a continuous load. The performances of the method suggested are compared with those of fuzzy logic and fuzzy-Proportional Integral (FL-PI) in term speed of convergence, of tracking time and tracking efficiency. The results of numerical simulation of our system confirmed the best performance of this method.
\end{abstract}

\section{Keywords}

MPPT, Sliding Mode-Fuzzy Logic Controller, Wind Energy Conversion System, Chattering

\section{Introduction}

Recently, the production of electrical energy by sources of renewable energy like the wind power increased because of the ecological problems and the increase in the cost of traditional energies and the limitation of their resources [1] [2] [3]. The power which a wind turbine can provide depends mainly on the atmos- 
pheric conditions; this is why it is necessary to carry out a system able to develop the maximum power for these various operating conditions [2]. As a result, we can have areas where we have a low variation in wind speed during a given period, which is why it is necessary to design an MPPT controller to perform in this kind of case.

Various MPPT controllers have been used for Wind Conversion Systems in previous publications such as Sliding Mode controller, thus the performances are reduce by the presence of the phenomenon of chattering [4] [5] [6]; Tip Speed Ratio controller, the first disadvantage of the ordering of the TSR is the measurement of wind speed, which is added to the cost of the system and present difficulties in the practical achievements, the second disadvantage is the need for obtaining the optimal value of TSR, which is different from one system to another [7]; Optimal Torque controller [8]; Power Signal Feedback controller, requires the knowledge of the curve of the maximum power of the wind turbine, requires of the manufacturer of the wind turbine of the tests of characterization (outside, blower) or of simulations of the profile of blades [9]; the algorithm Hill Climbing Search [10], the Increment Conductance method [11], the Perturb and Observe method [12] [13] because of the great non-linearity the wind speed, these techniques do not manage to follow the maximum power point and thus introduced strong fluctuations which involve a low power of exit; the Fuzzy Logic controller, can follow the non-linearity of the system and provides the best performance for the wind available, but with it only there is sometimes lack of adaptation and flexibility [2] [14] [15] and of many evolutionary algorithms [16] [17] [18] [19] [20]. These techniques differ in several aspects such as simplicity, speed, cost and effectiveness of convergence. More the share of these controllers MPPT are not performed for weak variations in wind speed and are unable to follow the maximum power point [17].

The objective of this manuscript is to realize a controller combining sliding mode and fuzzy logic in order to improve the maximum operation of a wind generator in areas where wind speeds are low.

The paper is organized as follows: the second section of the manuscript will be dedicated to the description of our study system. A third section is reserved for the SM-FL control strategy. The different simulations performed as well as the discussion of the results found will be the subject of the fourth section. In the fifth section, a conclusion is presented.

\section{System Modelling}

In this section, we present wind turbine model, Permanent Magnet Synchronous Generator and boost converter, which makes up the entire Wind Energy Conversion System, will be explained (Figure 1).

\subsection{Wind Turbine Modelling}

The aerodynamic power collected by a wind turbine is written in the following 


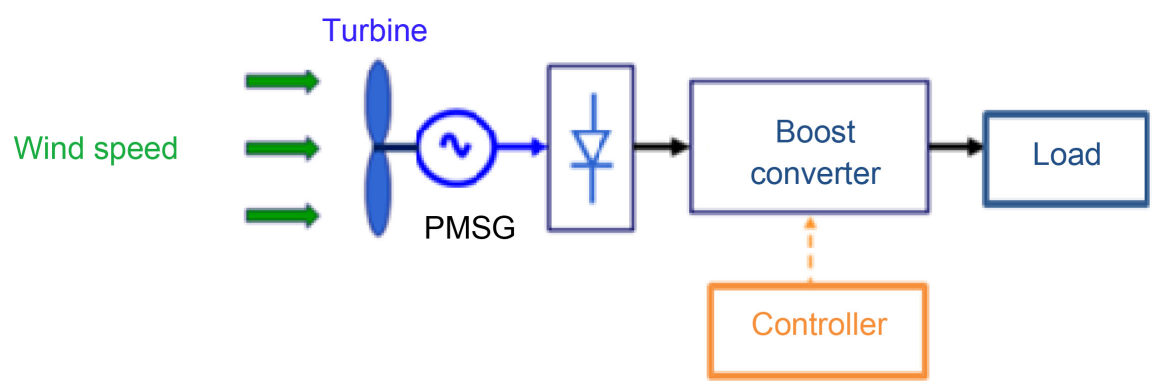

Figure 1. Structure of the study system.

form [15]:

$$
P_{\text {aero }}=\frac{1}{2} C_{p}(\lambda, \beta) \rho S V^{3}
$$

With:

$\rho$ : Represents the air density $\left(\mathrm{kg} / \mathrm{m}^{3}\right)$;

$V$ : wind speed $(\mathrm{m} / \mathrm{s})$;

$S$ : the useful surface crossed by the wind given by $S=\pi R^{2}$;

$R$ : the radius of the blades;

$C_{p}(\lambda, \beta)$ : Power coefficient.

The power coefficient $C_{p}(\lambda, \beta)$ indicates aerodynamic efficiency of wind turbine. It is a function of the speed $\lambda$ and the blade pitch angle $\beta$. It is different from one turbine to another due to its dependence on the characteristic of each. During this work, this coefficient will be modeled by the following analytical expression [19]:

$$
\begin{gathered}
C_{p}(\lambda, \beta)=0.5\left(\frac{98}{\lambda_{i}}-0.4 \beta-5\right) \exp \left(-16 / \lambda_{i}\right) \\
\frac{1}{\lambda_{i}}=\frac{1}{\lambda+0.08 \beta}-\frac{0.035}{\beta^{3}+1} \\
\lambda=\frac{R \omega_{m}}{V}
\end{gathered}
$$

The aerodynamic torque appearing at the level of the turbine is therefore a function of this power:

$$
\Gamma_{\text {aero }}=\frac{P_{\text {aero }}}{\omega_{m}}=\frac{1}{2 \omega_{m}} C_{p}(\lambda, \beta) \rho \pi R^{2} V^{3}
$$

where: $\omega_{m}$ is the rotor speed of a wind turbine. Table 1 presents the parameters of the wind turbine.

For each wind speed, it exists a maximum power of the wind turbine obtained according to the rotor speed (Figure 2).

\subsection{Permanent Magnet Synchronous Generator Modelling}

For applications autonomous of wind energy transformation, the PMSG are used the most considering their reliabilities and robustness. We used the referential $d-q$ 
Table 1. Wind turbine parameters.

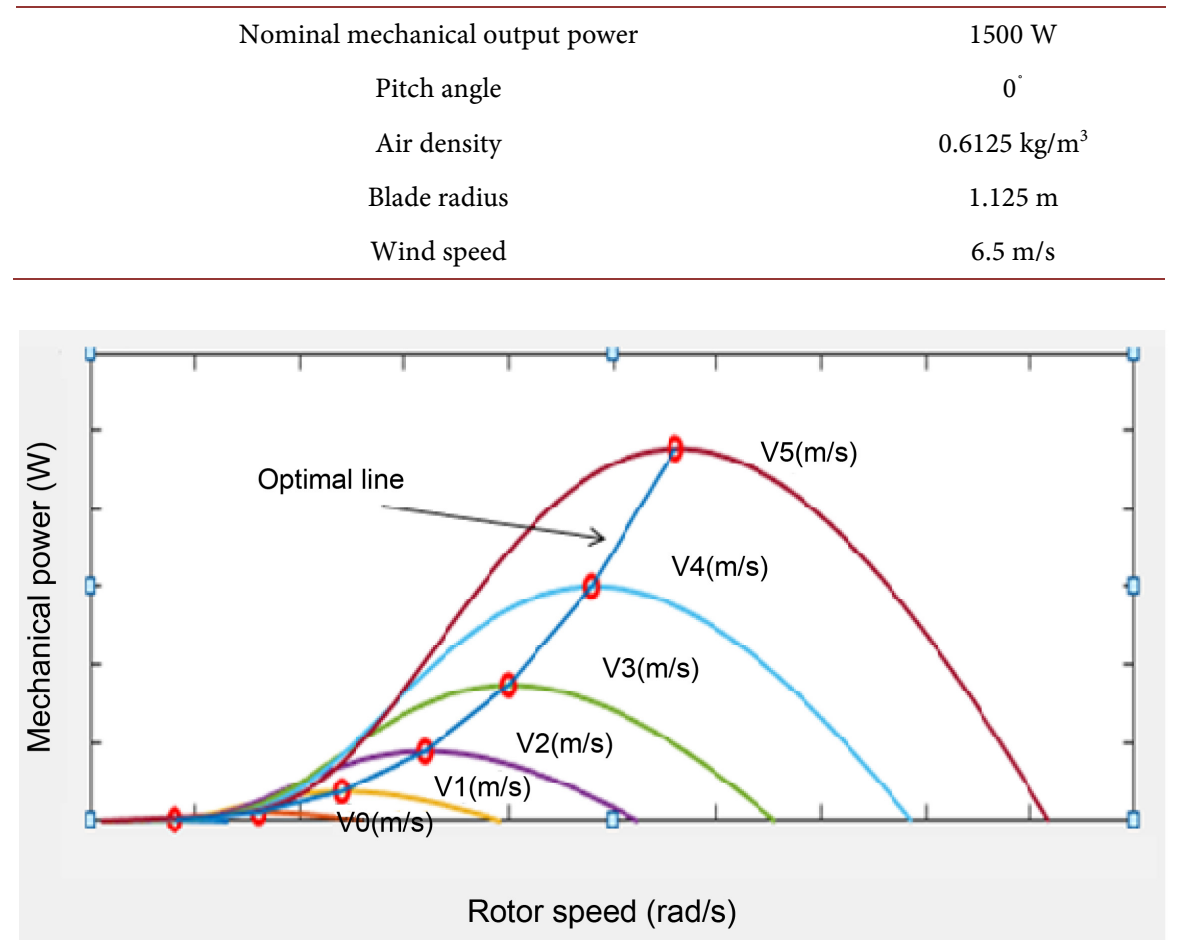

Figure 2. Maximum power of the wind turbine according to the rotor speed for different wind speed.

transform of park for modeling. The voltage of axis $d$ and $q$ is obtained by the system of Equation (6) [15].

$$
\left\{\begin{array}{l}
v_{d s}=R_{s} i_{d s}+L_{d} \frac{\mathrm{d} i_{d s}}{\mathrm{~d} t}-L_{q} \omega_{r} i_{q s} \\
v_{q s}=R_{s} i_{q s}+L_{q} \frac{\mathrm{d} i_{q s}}{\mathrm{~d} t}+L_{d} \omega_{r} i_{d s}+\omega_{r} \Psi_{r}
\end{array}\right.
$$

The Equation (7) gives the electromagnetic torque of PMSG.

$$
\Gamma_{e}=1.5 p\left[\left(L_{d}-L_{q}\right) i_{q s} i_{d s}-\Psi_{q r} i_{d r}-\Psi_{r} i_{q s}\right]
$$

with: $i_{d s}$ and $i_{q s}$ the currents of the axis $d$ and $q ; v_{d s}$ and $v_{q s}$ the voltages of the axis $d$ and $q$;

$\omega_{r}$ the angular frequency of generator;

$L_{q}$, and $L_{d}$ are the inductances of the generator;

$\Psi_{r}$ the permanent flux;

$R_{s}$ the stator resistance and $p$ is the pole pairs.

Table 2 recapitulates the parameters of the PMSG used for the numerical simulations.

\subsection{Modeling of Boost Converter}

Static converters are very significant for the wind energy transformation at variable speed. In this document, a boost converter is used here (Figure 3). During 
Table 2. PMSG parameters.

\begin{tabular}{cc}
\hline Rated power & $1500 \mathrm{~W}$ \\
Stator phase resistance & $0.425 \Omega$ \\
Machine inertia & $0.085 \mathrm{~kg} \cdot \mathrm{m}^{2}$ \\
Armature inductance & $0.225 \mathrm{H}$ \\
Friction factor & $0.00673 \mathrm{~N} \cdot \mathrm{m} / \mathrm{s}$ \\
Pole pairs & 5 \\
\hline
\end{tabular}

operation of the chopper, the switch is closed with a closing time equal to (D.T), and it is opened in an opening time $((1-D) . T)$, with: $T$ is the switching period and $D$ the duty cycle of the switch $(D \in[0,1])$.

$$
V_{\text {out }}=\frac{V_{\text {in }}}{1-D}
$$

where:

$$
\begin{aligned}
& V_{\text {out }} \text { : Output voltage; } \\
& V_{\text {in }} \text { : input voltage; } \\
& D \text { : duty cycle; }
\end{aligned}
$$

The boost converter parameters are summarized in Table 3.

\section{Controller Methods MPPT}

In this part, we initially will introduce the sliding mode (SM) controller, in continuation the fuzzy logic (FL) controller, and finally the combination of the two controllers (SM-FL).

\subsection{Sliding Mode Control (SM)}

The sliding mode control is one of the nonlinear controls effective. The design of this controller is carried out in three essential points: the establishment of the conditions of existence, choice of the sliding surfaces $(x)$, and the determination of the control law [6].

The maximum power point (MPP) condition is given by [21]:

$$
\frac{\mathrm{d} P_{W}}{\mathrm{~d} V_{W}}=0
$$

with $P_{w}, V_{w}, I_{w}$ is respectively the power, the voltage, and the current of the wind generator. The switching surface can be chosen as follows [20]:

$$
s(x, t)=\frac{\mathrm{d} P_{W}}{\mathrm{~d} V_{W}}=I_{W}+V_{W} \frac{\mathrm{d} I_{W}}{\mathrm{~d} V_{W}}
$$

Let $u$ be the switching control law defined according to the sign of the sliding surface adopted to increase and decrease the voltage $V_{W}$ according to the position of the operating point with respect to the PPM [6]:

$$
u=\left\{\begin{array}{l}
0 \text { for } s(x)>0 \\
1 \text { for } s(x)<0
\end{array} \text { Which can be written too: } u=\frac{1}{2}(1-\operatorname{sign}(S))\right.
$$




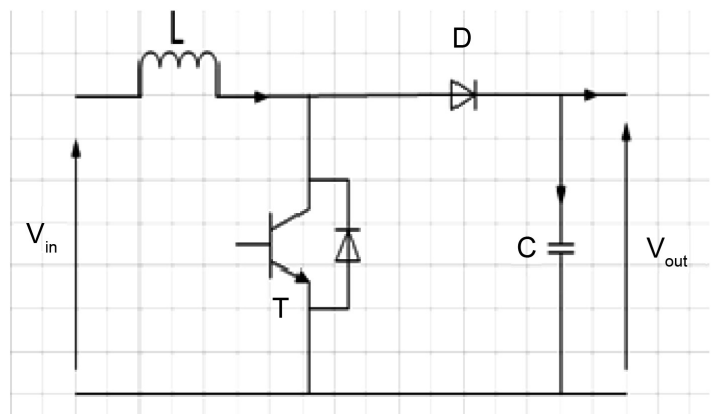

Figure 3. Boost converter.

Table 3. Boost converter parameters.

\begin{tabular}{cc}
\hline Load & $200 \Omega$ \\
Inductor & $3.635 \mathrm{mH}$ \\
Capacitor & $3.36 \mathrm{mF}$ \\
\hline
\end{tabular}

Equation (11) depends on the sign function. The use of this function in the control law makes it possible to force the error to converge asymptotically towards zero. However, this discontinuous function causes the reluctance problem. To remedy this problem in the literature either one applies the boundary layer solution where the sign function is approximated by the hyperbolic tangent function [22] [23], or one uses the higher order sliding mode command as a solution [24]. In our case we are going to add a fuzzy controller to the sliding mode exit; who, thanks to his membership functions, will be able to remedy this situation in another way.

\subsection{Fuzzy Logic Control (FL)}

The operation of this controller is done in three blocks: fuzzification, inference and defuzzification (Figure 4).

This controller is doing in three blocks: fuzzification, inference and defuzzification (Figure 4). Fuzzification converts the physical input variables into fuzzy sets. In our case, we have the error $E$ and the variation of the error $C E$ as inputs:

$$
\begin{aligned}
& E=\frac{P(k)-P(k-1)}{I(k)-I(k-1)} \\
& C E=E(k)-E(k-1)
\end{aligned}
$$

Linguistic variables are assigned to these quantities: NB (Negative Big), NM (Negative Medium), NS (Negative Small), Z (Zero), PS (Positive Small), PM (Positive Medium) and PB (Positive Big).

In the inference stage, we make decisions (Figure 5). Indeed, we establish logical relationships between the inputs and the output while defining the membership rules. Next, we draw up the table of inference rules (Table 4).

Finally, in defuzzification, we convert the fuzzy output subsets into a numeric value. 


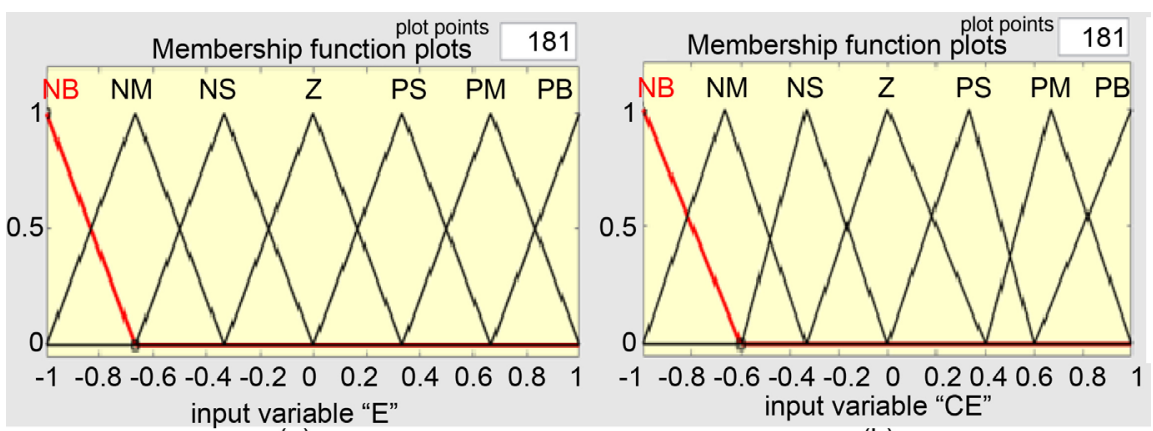

(a)

(b)

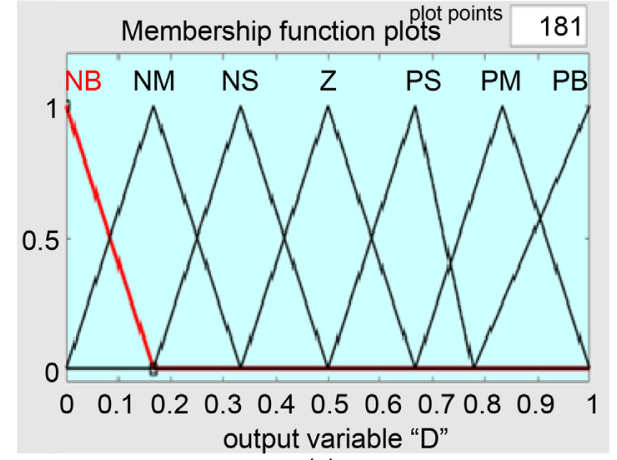

(c)

Figure 4. The sub-assemblies used (a), (b) and (c).

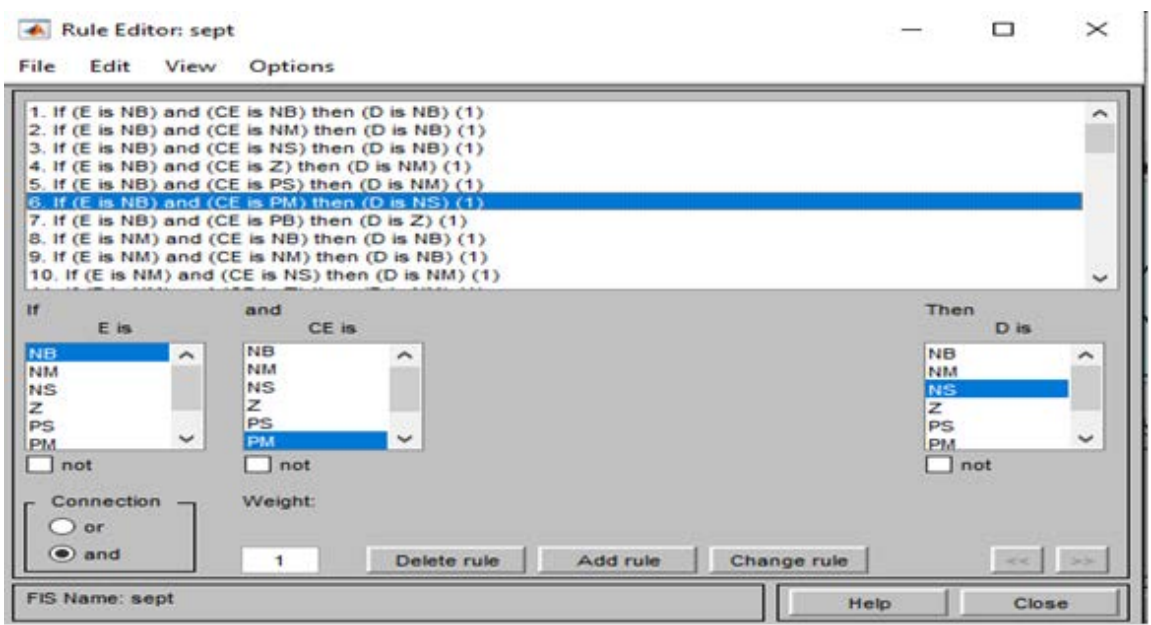

Figure 5. Rules inference according to Table 4.

Table 4. Table of inference rules.

\begin{tabular}{|c|c|c|c|c|c|c|c|}
\hline $\begin{array}{l}C E \\
\end{array}$ & NB & NM & NS & Z & PS & $\mathrm{PM}$ & PB \\
\hline NB & $\mathrm{NB}$ & NB & $\mathrm{NB}$ & NM & $\mathrm{NM}$ & NS & Z \\
\hline NM & $\mathrm{NB}$ & NB & $\mathrm{NM}$ & NM & NS & $\mathrm{Z}$ & PS \\
\hline NS & $\mathrm{NB}$ & $\mathrm{NM}$ & $\mathrm{NM}$ & NS & Z & PS & $\mathrm{PM}$ \\
\hline Z & NM & $\mathrm{NM}$ & NS & Z & PS & PM & PM \\
\hline PS & $\mathrm{NM}$ & NS & Z & PS & $\mathrm{PM}$ & $\mathrm{PM}$ & PB \\
\hline $\mathrm{PM}$ & NS & Z & PS & $\mathrm{PM}$ & $\mathrm{PM}$ & $\mathrm{PB}$ & PB \\
\hline PB & Z & PS & PM & $\mathrm{PM}$ & PB & PB & PB \\
\hline
\end{tabular}


After having realized our two controllers we can now combine them.

\subsection{Sliding Mode-Fuzzy Logic Control}

In this part, a SM-FL controller is designed in order to overcome the shortcomings of the SM. The SM controller is designed simply on the basis of Equation (10) and in order to have an SM-FL we add to the output of the SM a fuzzy controller and we obtain our structure which is given in Figure 6. The FL is recognized for its robustness against non-linear systems which reduce the chattering of the sliding mode and the SM is recognized for its rapid convergence which will readjust the dynamics of fuzzy rules. So the combination of these two SM-FL controllers makes it possible to have a controller that converges quickly and is robust.

\section{Results and Discussion}

After the text edit has been completed, the paper is ready for the template. Duplicate the In order to test the performance of the SM-FL MPPT controller, we have carried out several cases of simulations following two wind speed profiles of the wind turbine. To verify the theoretical study on the behavior of the MPPT controller, a series of simulations was carried out with the Matlab/Simulink software and a comparison was made with the controllers, fuzzy logic (FL) and fuzzy logic-PI (FL-PI). The gains of the PI controller used are: $\mathrm{Kp}=1.852$ and $\mathrm{Ki}=$ 1.338 .

Figure 7 and Figure 8 show the results obtained using our SM-FL approach and compared with FL and FL-PI. For the two profiles of wind (for example of 1.3 with 2.2s) our approach converges at the Maximum Point of Power with a

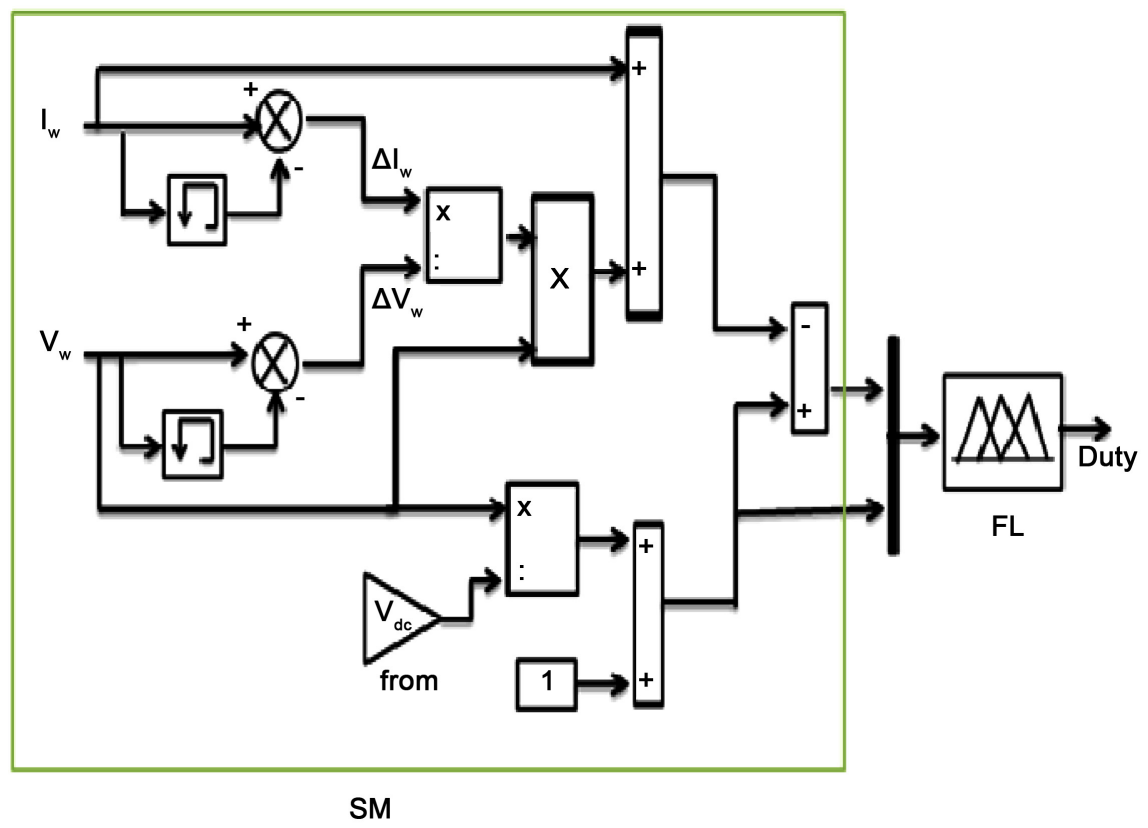

Figure 6. Design process of the hybrid method of MPPT developed. 


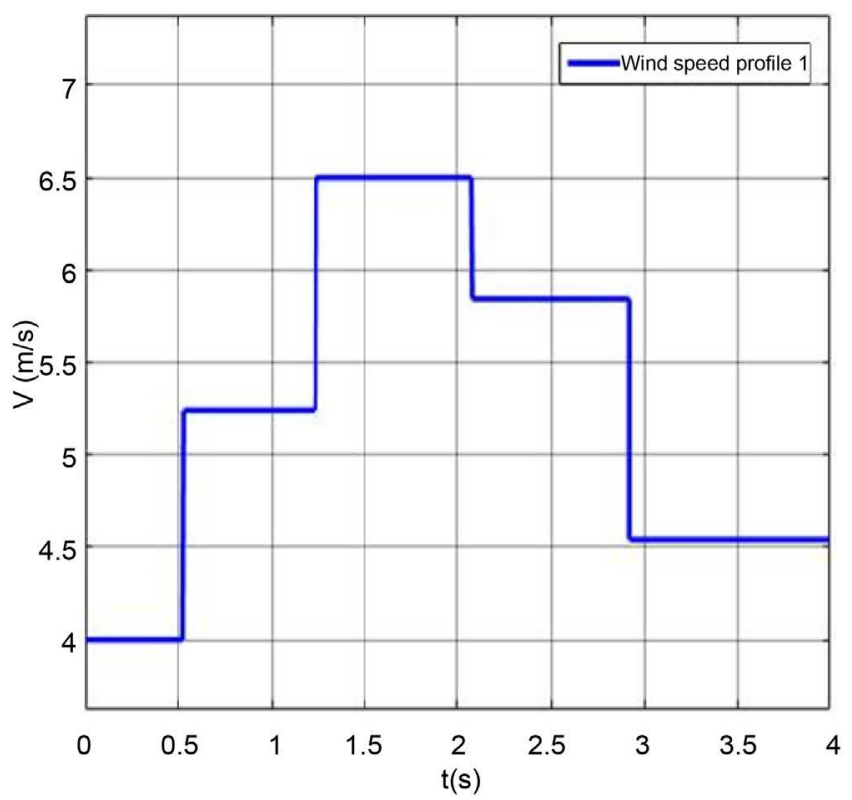

(a)

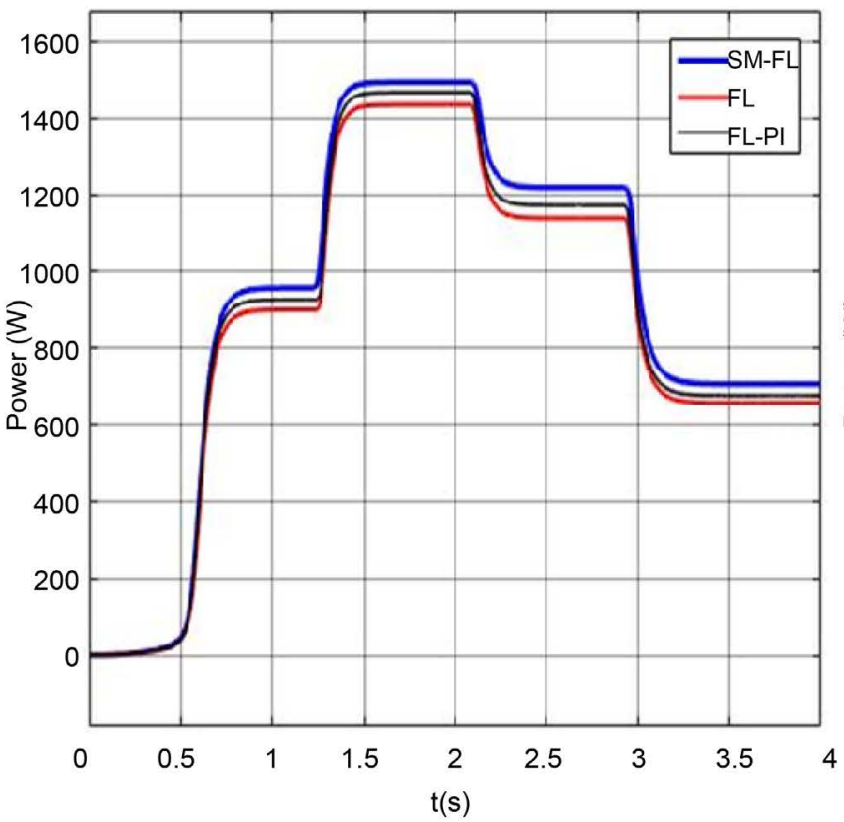

(c)

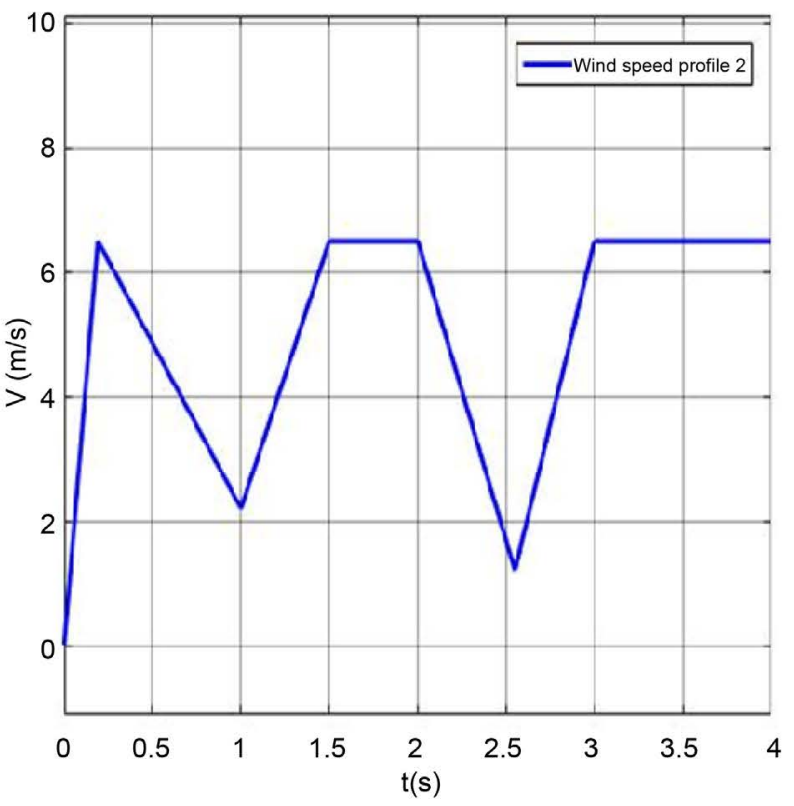

(b)

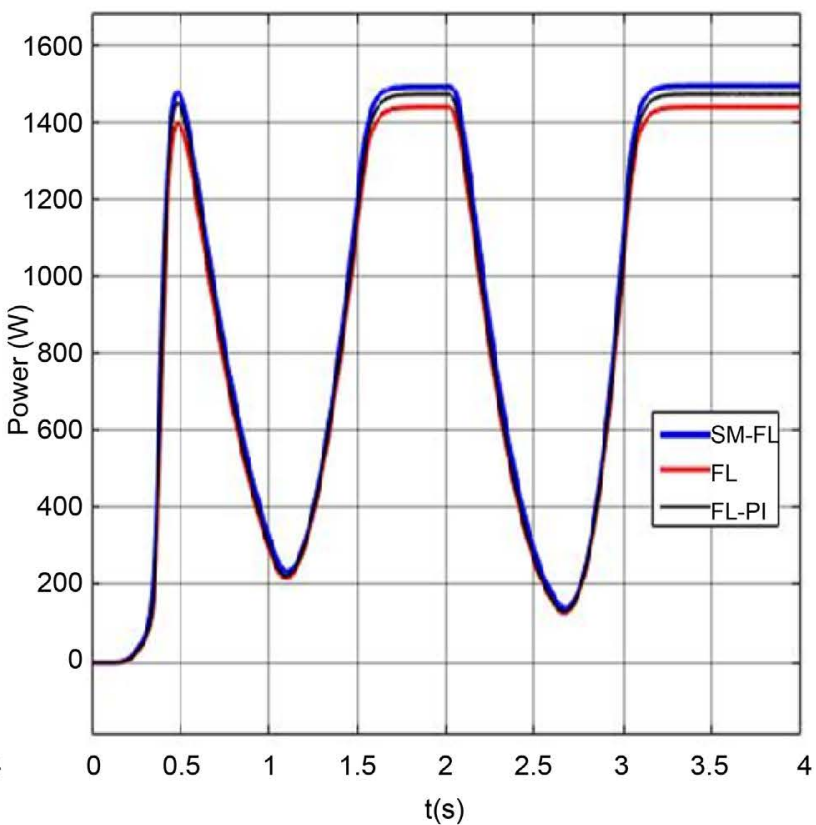

(d)

Figure 7. The powers obtained with the two wind profiles and their zoom.

fast response time, higher execution compared with both others during the changing of wind speed. For more details, the tracking efficiency is given in the table below. From these curves, we can see that the SM-FL controller contributed in a more efficient way to extract the maximum power for each wind speed by comparing to the MPPT strategies based on the FL and FL-PI approach. Because it is robust, it converges quickly compared to the compared approaches and it is very simple to achieve. Therefore, we can say that for low to medium wind ranges our controller is performing well. 
P. A. Malobe et al.

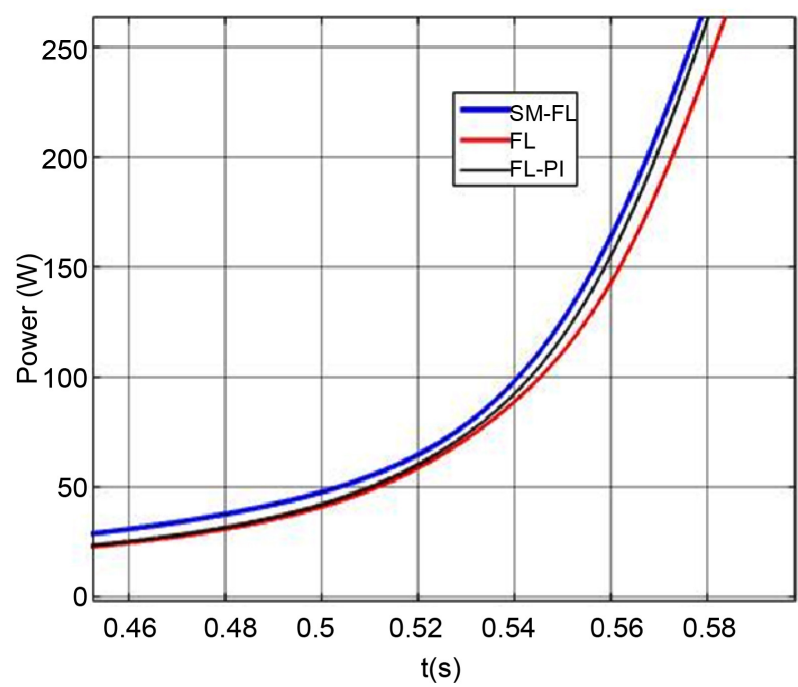

(a)

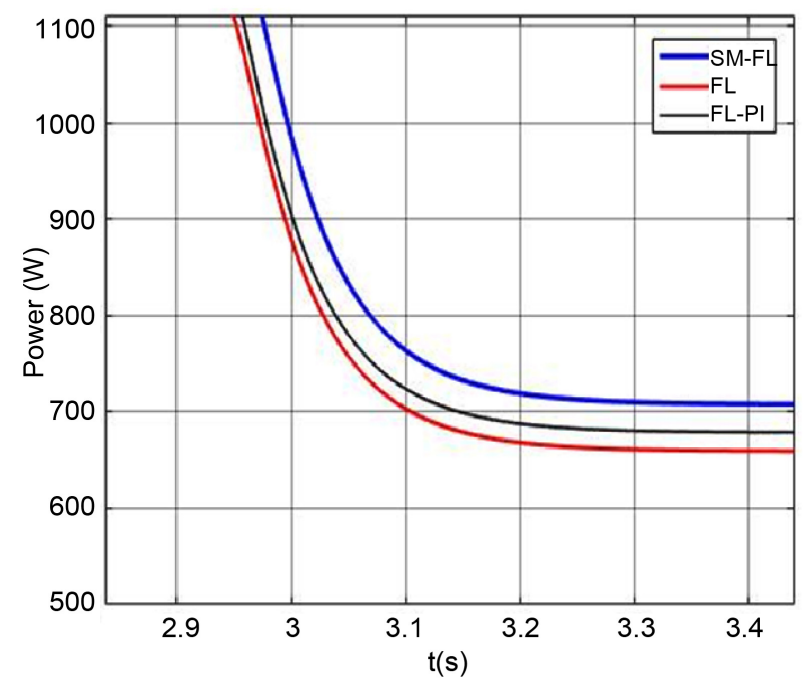

(c)

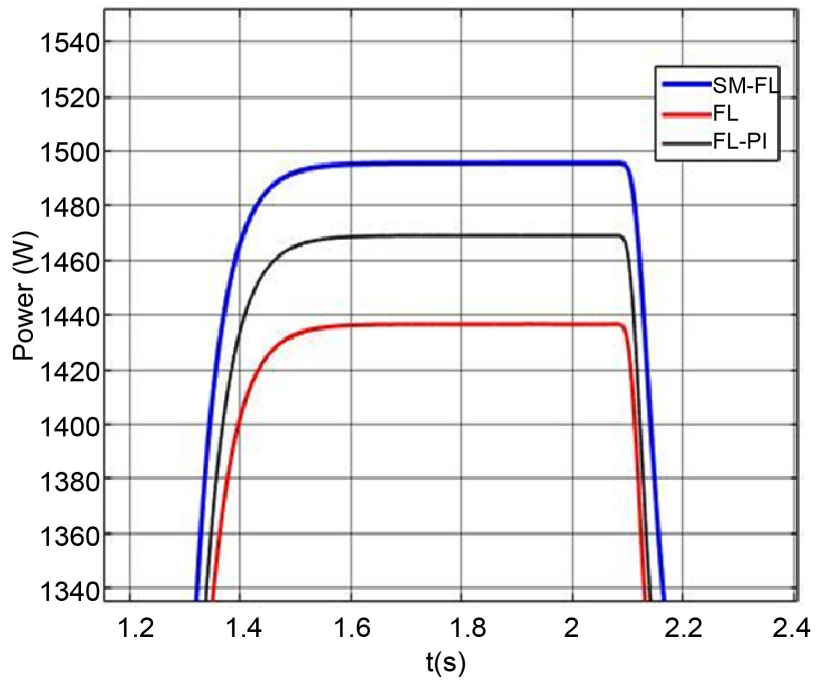

(e)

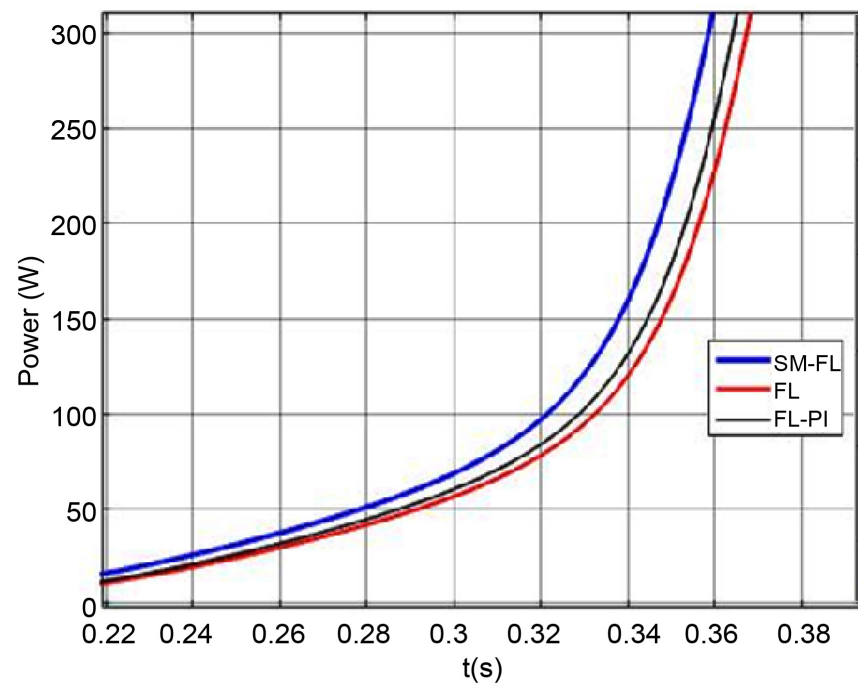

(b)

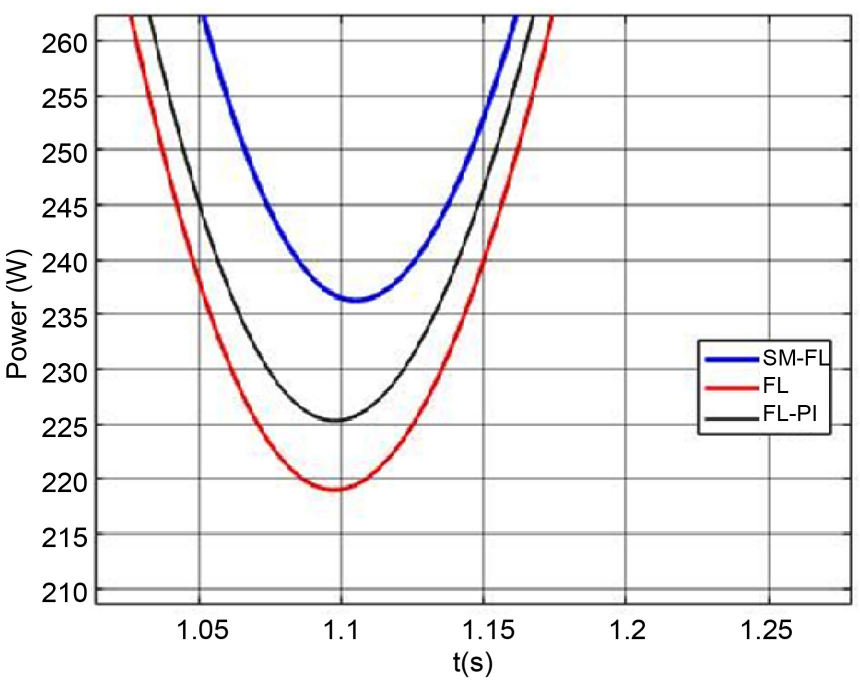

(d)

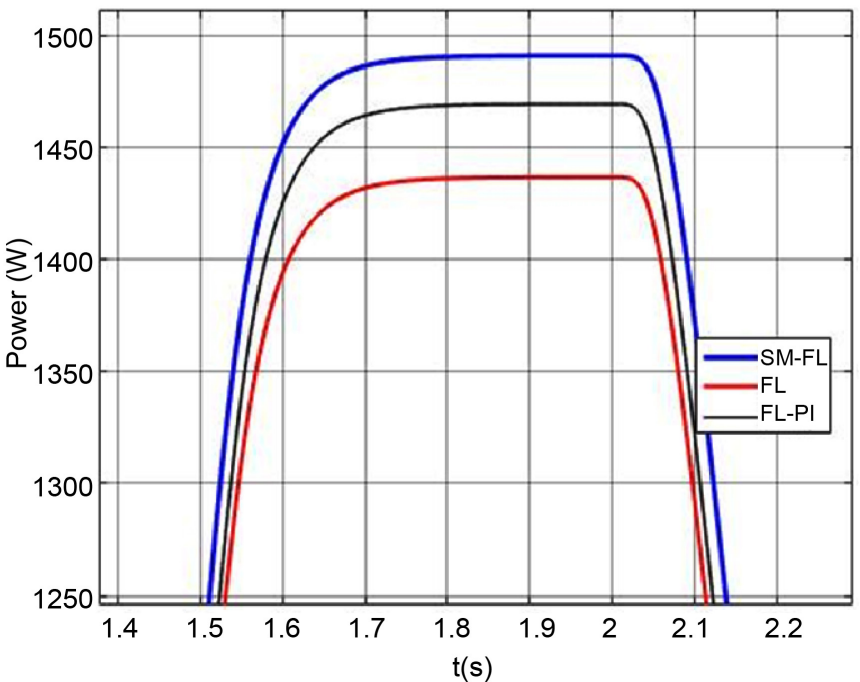

(f)

Figure 8. Zoom of powers. 


\begin{tabular}{ccccc}
\hline & \multirow{2}{*}{ Parameters } & \multicolumn{3}{c}{ Algorithms } \\
\cline { 3 - 5 } & & SM-FL & FL-PI & FL \\
\hline \multirow{2}{*}{ Wind } & Maximum power from the wind turbine (W) & 1500 & 1500 & 1500 \\
speed & Wind output power obtained (W) & 1496 & 1470 & 1438 \\
$6.5 \mathrm{~m} / \mathrm{s}$ & Tracking efficiency (\%) & 99.7 & 98 & 96 \\
\hline
\end{tabular}

\section{Conclusion}

With the aim of improving the efficiency of wind systems, especially their energy production in areas with low wind speeds; we have developed a smart and simple strategy, based on sliding mode and fuzzy logic. This strategy makes it possible to optimize the power delivered by the wind turbine for both wind speed profiles at all times. So we started with the presentation of the system used. Then we presented the SM and FL controllers. Then, we designed a sliding mode (SM) and fuzzy logic (FL) based controller, the SM-FL controller. The simulation results clearly demonstrate the effectiveness of the strategy adopted. Indeed, the MPPT SM-FL allowed on the one hand the pursuit of the maximum power point according to the weak variations of wind and on the other hand its performance compared to the FL and FL-PI approaches with its rapid convergence and its robustness.

\section{Acknowledgements}

The authors would like to thank the journal editor and all organizations that provided data for this research.

\section{Conflicts of Interest}

The authors declare no conflicts of interest regarding the publication of this paper.

\section{References}

[1] Erich, H. (2005) Wind Turbines: Fundamentals, Technologies, Application, Economics. 2nd Edition, Springer-Verlag, Berlin.

[2] Quang Minh, H., Frédéric, N., Najib, E. and Abdelaziz, H. (2012) Fuzzy Control of Variable Speed Wind Turbine Using Permanent Magnet Synchronous Machine for Stand-Alone System. In: M'Sirdi, N., et al., Eds., Sustainability in Energy and Buildings, Springer, Berlin, 31-44. https://doi.org/10.1007/978-3-642-27509-8_3

[3] Azizi, N. and CheshmehBeigi, H.M. (2017) Reactive and Active Power Control of Grid WECS Based on DFIG and Energy Storage System under Both Balanced and Unbalanced Grid Conditions. Journal of Renewable Energy and Environment, 4, 31-38.

[4] Hemdani, A., Dagbagi, M., Naouar, W.M., Idkhajine, L., Belkhodja, I.S. and Monmasson, E. (2015) Indirect Sliding Mode Power Control for Three Phase Grid Connected Power Converter. IET Power Electronics, 8, 977-985. https://doi.org/10.1049/iet-pel.2013.0945

[5] Nasiri, M., Mobayen, S. and Min Zhu, Q. (201) Super-Twisting Sliding Mode Control for Gearless PMSG-Based Wind Turbine. Complexity, 2019, Article ID: 
6141607. https://doi.org/10.1155/2019/6141607

[6] Spiazzi, G. and Mattavelli, P. (2002) Sliding-Mode Control of Switched-Mode Power Supplies. In: The Power Electronics Handbook, CRC Press, Boca Raton, Ch. 8. https://doi.org/10.1201/9781420037067.ch8

[7] Zhu, Y., Cheng, M., Hua, W. and Wang, W. (2012) A Novel Maximum Power Point Tracking Control for Permanent Magnet Direct Drive Wind Energy Conversion Systems. Energies, 5, 1398-1412. https://doi.org/10.3390/en5051398

[8] Kazmi, S.M.R., Goto, H., Guo, H. and Ichinokura, O. (2010) Review and Critical Analysis of the Research Papers Published Till Date on Maximum Power Point Tracking in Wind Energy Conversion System. IEEE Energy Conversion Congress and Exposition, Atlanta, 12-16 September 2010, 4075-4082.

https://doi.org/10.1109/ECCE.2010.5617747

[9] Barakati, S.M., Kazerani, M. and Aplevich, J.D. (2009) Maximum Power Tracking Control for a Wind Turbine System Including a Matrix Converter. IEEE Transaction Energy Conversion, 24, 705-713. https://doi.org/10.1109/TEC.2008.2005316

[10] Nateghi, A.R., Karegar, H.K. and Bagheri, S. (2014) Maximum Power Point Tracking of Permanent Magnet Wind Turbines Equipped with Direct Matrix Converter. Journal Renewable Sustainable Energy, 6, Article ID: 053123. https://doi.org/10.1063/1.4898365

[11] Radjai, T., Rahmani, L., Mekhilef, S. and Gaubert, J.P. (2015) Implementation of a Modified Incremental Conductance MPPT Algorithm with Direct Control Based on a Fuzzy Duty Cycle Change Estimator Using dSPACE. Solar Energy, 110, 325-337. https://doi.org/10.1016/j.solener.2014.09.014

[12] Ahmed, J. and Salam, Z. (2015) An Improved Perturb and Observe (P\&O) Maximum Power Point Tracking (MPPT) Algorithm for Higher Efficiency. Applied Energy, 150, 97-108. https://doi.org/10.1016/j.apenergy.2015.04.006

[13] Daili, Y., Gaubert, J.-P. and Rahmani, L. (2015) New Control Strategy for Fast-Efficient Maximum Power Point Tracking without Mechanical Sensors Applied to Small Wind Energy Conversion System. Journal Renewable Sustainable Energy, 7, Article ID: 043102. https://doi.org/10.1063/1.4923394

[14] Chen, Y.T., Jhang, Y.C. and Liang, R.H. (2016) A Fuzzy-Logic Based Auto-Scaling Variable Step-Size MPPT Method for PV Systems. Solar Energy, 126, 53-63. https://doi.org/10.1016/j.solener.2016.01.007

[15] Tiwari, R. and Babu, N.R. (2016) Fuzzy Logic Based MPPT for Permanent Magnet Synchronous Generator in Wind Energy Conversion System. IFAC-PapersOnLine, 49, 462-467. https://doi.org/10.1016/j.ifacol.2016.03.097

[16] Ro, K. and Choi, H. (2005) Application of Neural Network Controller for Maximum Power Extraction of Agrid-Connected Wind Turbine System. Electrical Engineering, 88, 53-45. https://doi.org/10.1007/s00202-004-0254-2

[17] Sabzevari, S., Karimpour, A., Monfared, M. and Sistani, M.B.N. (2017) MPPT Control of Wind Turbines by Direct Adaptive Fuzzy-PI Controller and Using ANNPSO Wind Speed Estimator. Journal Renewable Sustainable Energy, 9, Article ID: 013302. https://doi.org/10.1063/1.4973447

[18] Chiou, J.S. and Liu, M.T. (2009) Numerical Simulation for Fuzzy-PID Controllers and Helping EP Reproduction with PSO Hybrid Algorithm. Simulation Modelling Practice and Theory, 17, 1555-1565. https://doi.org/10.1016/j.simpat.2009.05.006

[19] Bouarroudj, N., Boukhetala, D. and Boudjema, F. (2014) Tuning Fuzzy PD ${ }^{\alpha}$ Sliding Mode Controller Using PSO Algorithm for Trajectory Tracking of a Chaotic System. Journal of Electrical Engineering, 14, 378-385. 
[20] Bouarroudj, N., Boukhetala, D. and Boudjema, F. (2015) A Hybrid Fuzzy Fractional Order PID Sliding-Mode Controller Design Using PSO Algorithm for Interconnected Nonlinear Systems. Journal of Control Engineering and Applied Informatics, 17, 41-51.

[21] Kermadi, M. and Berkouk, E.M. (2017) Artificial Intelligence-Based Maximum Power Point Tracking Controllers for Photovoltaic Systems: Comparative Study. Renewable and Sustainable Energy Reviews, 69, 369-386.

https://doi.org/10.1016/j.rser.2016.11.125

[22] Garraoui, R., Aroudi, A., Ben Hamed, M., Sbita, L. and Al-Hosani, K. (2016) A Comparative Study between Two MPPT Controllers Based on the Principe of Sliding-Mode Control Theory and Intelligent Control Technique in Photovoltaic Systems. In: Proceedings of the Mediterranean Conference on Information \& Communication Technologies 2015, Lecture Notes in Electrical Engineering, Vol. 380, Springer, Cham, 505-515. https://doi.org/10.1007/978-3-319-30301-7_53

[23] Yatimi, H. and Aroudam, E. (2016) Assessment and Control of a Photovoltaic Energy Storage System Based on the Robust Sliding Mode MPPT Controller. Solar Energy, 139, 557-568. https://doi.org/10.1007/978-3-319-30301-7_53

[24] Rashed, M., Goh, K.B., Dunnigan, M.W., MacConnell, P.F.A., Stronach, A.F. and Williams, B.W. (2005) Sensorless Second-Order Sliding-Mode Speed Control of a Voltage-Fed Induction-Motor Drive Using Nonlinear State Feedback. IEE Proceedings Electric Power Applications, 152, 1127-1136.

https://doi.org/10.1049/ip-epa:20050042 